\title{
An audit of seizure duration in electroconvulsive therapy
}

\author{
AIMS AND METHOD \\ Brief or missed seizures might \\ indicate that electronvulsive \\ therapy (ECT) is not being delivered \\ effectively. This cycle of audit at an \\ ECT clinic aimed to measure rates of \\ brief or missed seizures in two study \\ periods, before and after the acquisi- \\ tion of a more powerful ECT machine.
}

\author{
RESULTS \\ There was a significant reduction in \\ the rate of brief or missed \\ seizures in audit two. There was \\ a significant increase in the \\ restimulation rate in audit two, \\ but without the desired effect \\ of inducing adequate seizures.
}

\author{
CLINICAL IMPLICATIONS \\ Ineffective delivery of ECT might \\ result in poor response to the treat- \\ ment and should be minimised. \\ Certain aspects of ECT delivery \\ improved in the clinic studied but \\ some patients received an ineffective \\ second dose of electrical charge.
}

An influential audit of the practice of electroconvulsive therapy (ECT) in 1992 showed that in $22 \%$ of ECT treatments there was either no seizure or a brief seizure (defined as lasting 15 seconds or less). There was a perceived need for much clearer guidance and training in what to do on such occasions (Pippard, 1992). Clinical guidelines at the time recommended that restimulation at a given session should be carried out if a seizure of 15 seconds or less occurred (Royal College of Psychiatrists, 1989). This report describes a cycle of audit that aimed to monitor ECT practice in general, and seizure duration in particular, at the Crichton Royal Hospital, with reference to College guidelines.

\section{The study}

An initial audit (audit one) was performed in a 6-month period between August 1994 and January 1995. Trainees administering ECT had received training, based on College guidelines (Royal College of Psychiatrists, 1989), from the consultant responsible for the ECT clinic. They were encouraged to restimulate a patient with a higher charge (increase of 25 millicoulombs $(\mathrm{mC})$ ) in the event of a missed or brief seizure. Case notes and ECT record sheets of all patients receiving the treatment were examined and name, gender, age, diagnosis, concurrent medication, number of treatments per course, duration of motor seizure and course of action taken in the event of a brief seizure were noted.

A report was submitted to the trust's clinical audit committee and the ECT machine, an Ectron Series 5, was replaced with a more flexible and powerful machine, the Ectron Series 5A Ectonus. This accorded with the newly revised College guidelines (Royal College of Psychiatrists, 1995).

The audit was repeated (audit two) in a 6-month period between February 1999 and July 1999, using the method described above. Trainees were encouraged to adhere to the original ' 15 seconds or less' definition of a brief seizure to facilitate direct comparison of data from audit one. They were again advised to restimulate patients with missed or brief seizures with a higher charge (increase by one setting on the ECT machine). They were also asked to record seizure duration in the case notes, as well as on the ECT record sheet. This was to facilitate communication between the ECT clinic and the prescribing consultant, with particular reference to brief seizures, restimulation and stimulus alteration throughout a course of ECT.

\section{Results}

In audit one, 20 patients received 21 courses of ECT with a total of 165 treatments. In audit two, 23 patients received 27 courses, with a total of 169 treatments. With a catchment population of 150000 and extrapolating to a whole year, these figures give annual averages of 2.2 and 2.3 treatments per 1000 population in audit one and audit two, respectively. These figures are close to the national average for Scotland (Robertson et al, 1997). Demographic data and the mean numbers of treatments per course are shown in Table 1. Each course of ECT is counted, giving $n$ of 21 and 27, respectively.

Most patients in both audit periods were depressed, 19 of $21(91 \%)$ in audit one and 17 of $27(63 \%)$ in audit two, with no statistically significant differences. The two other patients in audit one had diagnoses of schizoaffective disorder and schizophrenia, respectively, and in audit two, six patients had schizoaffective disorder, two had schizophrenia and two had mania.

Tricyclic antidepressants (TCAs) (including trazodone and lofepramine) were prescribed at a significantly lower rate in audit two (Yates-corrected $\chi^{2}=4.714, P=0.006$, d.f.=1), with a swing towards prescribing newer antidepressants such as venlafaxine, nefazodone and mirtazapine. There were no differences between the use of

Table 1. Gender and age of patients and number of courses of ECT

\begin{tabular}{|c|c|c|}
\hline & Audit one & Audit two \\
\hline Male (\%) & $2 \quad(9.5)$ & $11(40.7)^{1}$ \\
\hline Female (\%) & 19 (90.5) & $16(59.3)$ \\
\hline Mean age (s.d.) & $58.1(16.7)$ & 54.7 (15.5) Ns ( $t$ test) \\
\hline $\begin{array}{l}\text { Mean number of } \\
\text { courses (s.d.) }\end{array}$ & $7.7 \quad(4.5)$ & $6.3(2.6) \mathrm{NS}(t$ test) \\
\hline
\end{tabular}

1. Yates-corrected $\chi^{2}=4.355, P=0.04$, d.f. $=1$. 
minor tranquillisers, such as benzodiazepines and zopiclone, antipsychotics, lithium or anticonvulsants. Seizure durations are shown in Table 2.

'Adequate' seizures (more than 15 seconds) were achieved more commonly in audit two (Yatescorrected $\chi^{2}=4.01$, d.f. $=1, P<0.05$ ).

The course of action taken when the seizure was brief differed markedly between audits. In audit one, 4 out of $35(11 \%)$ of brief seizures were followed with restimulation with an increased dose compared with 16 of $21(76 \%)$ in audit two (Yates-corrected $\chi^{2}=21.24$, d.f. $=1, P<0.0001)$. However, in audit one, 2 of 4 and in audit two, 4 of 16 restimulations with an increased dose resulted in an adequate seizure. Furthermore, in audit two, 7 of 36 seizures lasting 16 to 24 seconds were erroneously followed by restimulation, with only 2 adequate seizures occurring as a result. Thus, the total number of restimulations, regardless of whether the initial seizure was 'adequate', was 27 . Only 8 of these restimulations resulted in adequate seizures, a 'success' rate of $30 \%$.

\section{Discussion}

The importance of monitoring seizure activity has been emphasised (Pippard, 1992; Royal College of Psychiatrists, 1995) but the method varies widely from electroencephalogram (EEG) monitoring to monitoring of seizure activity, with or without the Hamilton cuff technique (Adderley \& Hamilton, 1953). A recent Indian study suggested that monitoring of motor seizure is unreliable, with $7 \%$ of EEG-adequate seizures deemed inadequate according to this measure (Mayur et al, 1999). Monitoring without the cuff technique is the commonest method (Robertson et al, 1997; Duffett \& Lelliott, 1998) and is used in the ECT clinic studies here. The definition of a brief seizure (less than 15 seconds) is arbitrary and the relevance of duration to clinical outcome has been questioned (Fear et al, 1994). It should not be taken as the sole marker of the adequacy of treatment (Pippard, 1992; Royal College of Psychiatrists, 1995). However, it remains an important parameter of ECT treatment, as evidenced by the recent finding that patients with 'inadequate' seizures had a lower response rate to ECT (Krystal et al, 2000), and my report shows some interesting results and issues for debate.

First, the proportion of brief seizures has decreased significantly in this clinic. Several factors might account for this, including age, gender, medication, type of ECT machine and operator training. There were more males in audit two but, as male gender is associated with a higher

\section{Table 2. Seizure duration and need for restimulation}

\begin{tabular}{|lcc}
\hline & $\begin{array}{c}\text { Audit 1 } \\
n=165\end{array}$ & $\begin{array}{c}\text { Audit 2 } \\
n=169\end{array}$ \\
\hline $\begin{array}{c}\text { No need for restimulation (16 seconds or } \\
\text { more) }\end{array}$ & 130 & 148 \\
$\begin{array}{l}\text { Need for restimulation (15 seconds or less) } \\
\text { need }\end{array}$ & 35 & 21
\end{tabular}

seizure threshold, this might be expected to increase the rates of brief seizures. TCA use has diminished, as reported in an earlier audit (Tresize, 1998), but the effects of TCAs and selective serotonin reuptake inhibitors, which both reduce the seizure threshold, are complex and probably vary between members of each drug group (Royal College of Psychiatrists, 1995). Therefore, the change in prescription of antidepressants may not be relevant. It is noteworthy that benzodiazepine use has not changed.

The clinic updated its machine to the Ectron Series $5 \mathrm{~A}$ after audit one. This allowed greater dosing flexibility, with an output range of $25-700 \mathrm{mC}$ compared with the older machine which had a maximum output of $400 \mathrm{mC}$. It is interesting that in audit one, 34 of the brief seizures occurred in one patient, a 65-year-old female on a low dose of diazepam, despite using $400 \mathrm{mC}$ in most of the treatments. In audit two, 19\% of the brief seizures occurred in one patient, a 51-year-old male who required diazepam in very high doses. He received increasing doses (up to $700 \mathrm{mC}$ ) with a concomitant increase in seizure duration. He would probably have had much shorter seizures with the older, less powerful machine. It is also possible that improved operator training had an effect, particularly the greater recognition of the importance of increasing the dose throughout a course of ECT if seizure duration decreases (Robertson et al, 1995; Royal College of Psychiatrists, 1995). Improved documentation and communication between the ECT clinic and ward teams facilitated dose increases.

The second major finding is the disappointingly low 'success' rate of restimulation. Trainees followed guidelines in the event of a brief seizure more often in audit two but the induction of an adequate seizure only occurred in $25 \%$ of cases. Many factors might contribute to this but it may be that the patient is restimulated too quickly without allowing the raised, post-ECT seizure threshold to drop. In a study of multiple ECT in open session, the period between stimulations was 3 minutes (Roemer et al, 1990), a much longer period than that used in routine practice.

\section{Conclusion}

This report has demonstrated that an improved rate of adequate seizures has been achieved, probably with use of a more flexible machine and with improved operator training and communication between the ECT clinic and the prescribing consultant. An increased rate of restimulation after brief seizures has been achieved but with a low rate of seizure induction. This is an area of concern given that patients, often elderly, receive ineffective additional stimulation with the risk of increased posttreatment cognitive impairment and other side-effects. Perhaps this group should not be restimulated, especially if they are showing clinical improvement?

To further improve the practice of ECT in this clinic, the use of stimulus titration should be considered, with or without EEG monitoring (Royal College of Psychiatrists, 1995). Coupled with dose increases throughout a course 
of ECT, this should further reduce the rate of brief seizures. If patients are to be restimulated, sufficient time should be allowed for the seizure threshold to drop and a greater dose increment than that currently employed should be considered.

Further audit should be carried out to evaluate the effects of such measures.

\section{Declaration of interest}

None.

\section{References}

ADDERLEY, D. J. \& HAMILTON, M. (1953) Use of succinylcholine in ECT. British Medical Journal, i, 195-197.

DUFFETT, R. \& LELLIOTT, P. (1998) Auditing electroconvulsive therapy. The third cycle. British Journal of Psychiatry, $172,401-405$.
FEAR, C. F., LITTLEJOHNS, C. S., ROUSE, E., et al (1994) Propofol anaesthesia in electroconvulsive therapy. Reduced seizure duration may not be relevant. British Journal of Psychiatry, 165 506-509.

KRYSTAL, A. D., DEAN, M. D., WEINER,
R. D., et al (2000) ECTstimulus intensity: are present ECTdevices too limited? American Journal of Psychiatry, 157, 963-967.

MAYUR, P. M., GANGADHAR, B. N., JANAKIRAMAIAH, N., et al (1999)

Motor seizure monitoring during electroconvulsive therapy. British Journal of Psychiatry, 174, 270-272.

PIPPARD, J. (1992) Audit of electroconvulsive treatment in two National Health Service regions. British Journal of Psychiatry, 160, 621-637. ROBERTSON, C., WHEELDON, T., EAGLES, J. M., et al (1995) Improving electroconvulsive therapy practice through audit. Psychiatric Bulletin, 19, $480-481$.

- FREEMAN, C. P. L. \& FERGUSON, G. (1997) ECT in Scotland. Psychiatric Bulletin, 21, 699-702.

Tom MacEwan Consultant in Old Age Psychiatry, Grampian Primary Care NHS Trust, Mental Health Service, Clerkseat Building, Royal Cornhill Hospital, Cornhill Road, Aberdeen AB25 2ZH et al. $473-478$
ROYAL COLLEGE OF PSYCHIATRISTS

(1989) The Practical Administration of ElectroconvulsiveTherapy (ECT). London: Gaskell.

— (1995) The ECT Handbook. Council Report CR39. London: Royal College of Psychiatrists.

TREZISE, K. (1998) Changes in practice of ECT: a follow-on study. Psychiatric Bulletin, 22,687-690.
ROEMER, R. A., DUBIN,W. R., JAFFE, R., et al (1990) An efficacy study of

single- versus double-seizure induction with ECT in major depression. Journal of Clinical Psychiatry, 51,

original papers

Psychiatric Bulletin (2002), 26, 339-341

\section{AILEEN O'BRIEN AND MIKE FIRN}

\section{Clozapine initiation in the community}

\section{AIMS AND METHOD}

The study aims to describe

the experience of an assertive community treatment team when commencing clozapine at home rather than in hospital, following a locally-devised policy. Any failed

\author{
attempts and problems experienced \\ are described.
}

RESULTS

There have been no serious adverse events with 13 patients who have been started on clozapine at home.

\section{CLINICAL IMPLICATIONS}

For an assertive community treatment team, home-initiation of clozapine is a practical option, particularly when patients refuse to come in to hospital.
Clozapine has been established as an effective therapy for treatment-resistant psychosis (Kane et al, 1988). It has been shown to have benefits in terms of quality of life and reduction of both positive and negative symptoms (Grace et al, 1996; Rosenheck et al, 1997), and it is cost-effective in the long term because of the reduction in hospital admissions (Aitchison \& Kerwin, 1997; Percudani et al, 1999).

However, it is not without its problems, the main ones being the requirement for regular monitoring through blood tests and side-effects such as drowsiness and weight gain. Another drawback is the traditional prerequisite that all patients being started on clozapine need to come into hospital. Difficulties arise in encouraging a patient with schizophrenia to take medication in the first instance. When you consider the blood tests and the requirement for hospital admission, it is understandable that clozapine is underused in a population who, potentially, would greatly benefit from it.
This issue is especially relevant to an assertive community treatment (ACT) team, where patients with severe mental illness are specifically referred with characteristics of being difficult to engage and having poor compliance with treatment. Such patients are likely to have negative attitudes towards hospital admission. The ACT Model (Stein \& Test, 1980) is built around providing home-based alternatives to hospital admission for 'revolving door' patients with psychoses.

The pharmaceutical company that operates the Clozari ${ }^{\circledR}$ Patient Monitoring Service (CPMS) recommends hospital admission because of the risk of hypotension, excessive sedation and fits. In the USA, initiating clozapine in a partial hospitalisation programme (Johnson et al, 1994) has been reported as both safe and successful.

In our ACT team based in Wandsworth, south London, we have been starting clozapine in the community, rather than in hospital, since 1995. Accordingly, we have set out to evaluate the initiation of clozapine at home in a naturalistic, uncontrolled clinical setting. 